\title{
Depressão e exposição aos agrotóxicos em pequenas agricultoras no oeste do Paraná
}

\section{Depression and exposure to pesticides in small farmers in western Paraná}

Nathália Nascimento Reis ${ }^{1}$, Gicelle Galvan Machineski², Manoela de Carvalho ${ }^{3}$, Maria Lúcia Frizon Rizzotto 4

1. ORCID: https://orcid.org/0000-0002-0756-914X. Enfermeira. Universidade Estadual do Oeste do Paraná, Cascavel, Paraná, Brasil. E-mail: reisnathalia@hotmail.com

2. ORCID: https://orcid.org/0000-0002-8084-921X. Doutora em Enfermagem. Universidade Estadual do Oeste do Paraná, Cascavel, Paraná, Brasil. E-mail: gmachineski@gmail.com

3. ORCID: https://orcid.org/0000-0003-4226-1332. Doutora em Enfermagem. Universidade Estadual do Oeste do Paraná, Cascavel, Paraná, Brasil. E-mail: man0ela@hotmail.com

4. ORCID: https://orcid.org/0000-0003-3152-1362. Doutoraem SaúdeColetiva. Universidade Estadual do Oeste do Paraná, Cascavel, Paraná, Brasil. E-mail: frizon@terra.com.br

CONTATO: Autor correspondente: Gicelle Galvan Machineski | Endereço: Rua Universitária, 1619. Jardim Universitário - CEP: 85819-110 - Cascavel - PR, Brasil. Telefone: (45)999810684. E-mail: gmachineski@gmail.com

RESUMO Apesar de ser uma doença multifatorial, estudos indicam que a depressão pode estar associada a exposição a agrotóxicos. Objetivou-se descrever o perfil, aspectos de vida, trabalho e adoecimento de mulheres depressivas expostas a agrotóxicos em um município localizado no oeste do Paraná. Trata-se de pesquisa descritiva, qualitativa com 10 mulheres selecionadas a partir de inquérito populacional, por meio de entrevista com uma questão norteadora, no período de março a abril de 2017. As informações foram submetidas à análise temática. As mulheres tinham entre 18 e 65 anos de idade, baixa escolaridade e estavam inseridas no mercado de trabalho. A partir da análise das informações identificaram-se as seguintes temáticas: Início da vida laboral na infância, exposição a agrotóxicos durante longo período da vida sem proteção, aspectos da vida e do trabalho, história de exposição e intoxicação 
por agrotóxicos e; adoecimento por depressão. Sugerem-se estudos epidemiológicos e clínicos complementares sobre a relação desse fenômeno com o desenvolvimento de transtornos de humor.

DESCRITORES: Agrotóxicos. Saúde mental. Saúde Coletiva.

ABSTRACT Despite being a multifactorial disease, studies indicate that depression may be associated with exposure to pesticides. The objective was to describe the profile, aspects of life, work and illness of depressed women exposed to pesticides in a municipality located in the west of Paraná. This is a descriptive, qualitative research with 10 women selected from a population survey, through an interview with a guiding question, from March to April 2017. The information was submitted to thematic analysis. The women were between 18 and 65 years of age, with low education and were included in the labor market. From the information analysis, the following themes were identified: Beginning of working life in childhood, exposure to pesticides for a long life period without protection, life and work aspects, history of exposure and poisoning by pesticides and; illness due to depression. Complementary epidemiological and clinical studies are suggested on the relationship of this phenomenon with the development of mood disorders.

DESCRIPTORS: Agrochemicals. Mental Health. Public Health. 


\section{INTRODUÇÃO}

o

Brasil se apresenta desde 2008 como um dos maiores consumidores de agrotóxicos do mundo e a região oeste do estado do Paraná, era a terceira região do estado que mais consumia agrotóxicos no ano de $2013^{1-2}$. O município, onde realizou-se o estudo, apresenta como principal atividade econômica a produção agrícola e entre os anos de 2013 a 2015 comercializou 289,6 toneladas de agrotóxicos ${ }^{3}$.

Devido ao grau de toxicidade apresentado pelas substâncias agrotóxicas, os efeitos nocivos gerados na saúde da população variam de acordo com o grau e a forma de exposição, a quantidade absorvida, e o princípio ativo de cada substância. Entre os principais impactos na saúde destacam-se alergias, os distúrbios gastrointestinais, respiratórios, endócrinos, neurológicos e reprodutivos, neoplasias, mortes acidentais e o suicídio ${ }^{4}$

O uso de agrotóxicos pode provocar intoxicações classificadas como agudas, subagudas e crônicas 5 . As formas de intoxicações acontecem de acordo com a exposição, independente da maneira como ocorrem, seja intencional, acidental, ocupacional ou ambiental ${ }^{6}$

As intoxicações crônicas podem resultar em problemas de saúde mental e grande parte dos casos de intoxicações notificados são relacionados à tentativa de suicídio. $O$ estado do Paraná é o primeiro colocado no país em número de notificação e tentativas de suicídio, dos 3.723 casos notificados por intoxicação em 2017, cerca de 1.631 são relacionados a tentativa de suicídio, o que corresponde a um percentual de $40 \%{ }^{7}$.

Estudos realizados em diversos países apontam a relação entre a exposição a agrotóxicos e o desenvolvimento de depressão, isso porque essas substâncias atuam nos sistemas de comunicação cerebral, diminuindo a disponibilidade de neurotransmissores como acetilcolina e serotonina, o que contribui para o desenvolvimento de transtornos mentais, especialmente a depressão ${ }^{8-10}$.

Os pesticidas, organofosforados e carbamatos foram identificados como inibidores da atividade da acetilcolinesterase, sendo associados ao desenvolvimento de depressão. Aumentando os níveis de acetilcolina em até $15 \%$ nos períodos em que a exposição à agrotóxicos é mais intensa? ${ }^{9}$

Em estudo realizado no Brasil, no estado do Rio Grande do Sul, identificou que a exposição aos agrotóxicos pode aumentar em cerca de 50\% a chance para o desenvolvimento de transtornos mentais menores e que a exposição a essas substâncias é cumulativa, sendo considerada condição que predispõe o desenvolvimento de depressão. Além disso, as pessoas expostas aos agrotóxicos podem ter até 2,5 vezes mais chances de apresentaram algum tipo de problema neurológico do que as pessoas que não tiveram nenhum contato com substâncias agrotóxicas ${ }^{10}$. 
Dados publicados pela World Health Organization (WHO) ${ }^{11}$ indicam que aproximadamente 322 milhões de pessoas vivem com depressão no mundo. E estudos tem relacionado o desenvolvimento da doença e da ideação suicida com a exposição aos agrotóxicos ${ }^{8,12}$.

A partir de inquérito populacional a respeito da exposição a agrotóxicos identificou-se que a maioria, $75 \%$ dos 624 indivíduos que participaram do estudo e que relatou quadro depressivo, era do sexo feminino. Nesse sentido, questiona-se: "Como se caracterizam o perfil, aspectos de vida, trabalho e adoecimento de mulheres com diagnóstico de depressão expostas a agrotóxicos?". Desse modo o presente estudo teve por objetivo descrever o perfil e aspectos de vida, trabalho e adoecimento de mulheres depressivas expostas a agrotóxicos em um município localizado na região Oeste do estado do Paraná.

\section{MÉTODO}

Trata-se de pesquisa descritiva, qualitativa que faz parte do projeto intitulado "Avaliação de Saúde de populações expostas a agrotóxicos na região Oeste do Estado do Paraná" aprovado pelo CEP com o parecer n 1.696.960/2016.

Nesse projeto, foi realizado um inquérito no município de Anahy, a partir de uma ficha ampliada do Protocolo de Investigação de populações expostas aos agrotóxicos do estado do Paraná (constituída de dados sobre escolaridade; trabalho; renda; caracterização do contato com os agrotóxicos, nomes dos produtos, tempo de exposição, descrição da atividade geradora da exposição, intoxicações pregressas e; história ocupacional), aplicada a um membro de cada uma das 230 famílias participantes, no período de março a abril de 2017.

Para o presente estudo, a coleta das informações foi realizada a partir de entrevista com dez mulheres, escolhidas intencionalmente, que estavam em tratamento para depressão e relataram exposição direta ou indireta aos agrotóxicos no referido inquérito, com diagnóstico de depressão registrado em prontuário da unidade básica de saúde do município. Realizou-se a coleta das informações até que se alcançou a saturação em que se atingiu o objetivo do estudo, conforme observado na Figura 1. 
Figura 1. Fluxograma da coleta de dados

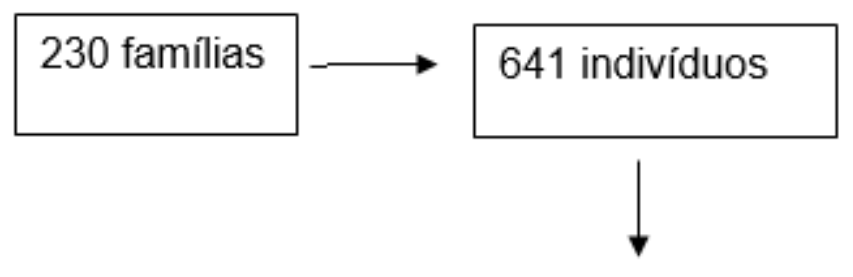

Escolha intencional de indivíduos que estavam em tratamento para depressão. E coleta de informações até o alcance da saturação

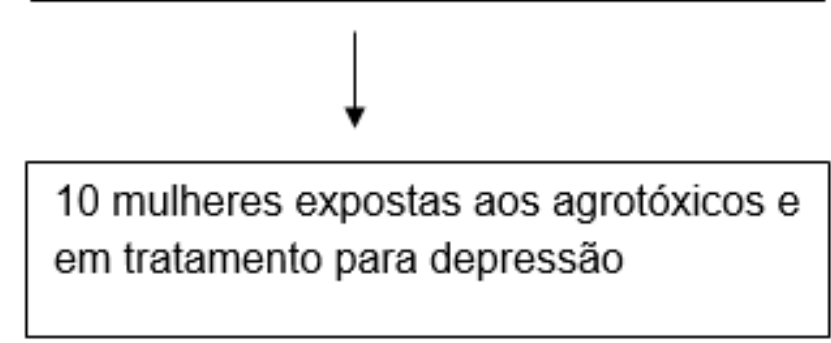

Fonte: Elaborado pelas autoras (2020).

A entrevista com as mulheres selecionadas teve a seguinte questão norteadora: "Conte-me sobre a sua história de vida e de trabalho", que contemplou um roteiro sobre o perfil, os aspectos de vida, trabalho e adoecimento. As informações foram gravadas e após transcritas na íntegra, identificadas pela letra $\mathrm{E}$ seguido do número da ordem em que aconteceram as entrevistas, sendo submetidas a análise temática em que se realizou leitura flutuante, leitura aprofundada em busca de significados e análise e tratamento das informações para a elaboração de unidades temáticas ${ }^{12-13}$.

\section{RESULTADOS}

A partir da análise dos resultados foram descritas as unidades temáticas referentes aos aspectos da vida e do trabalho, à história de exposição e intoxicações por agrotóxicos e ao adoecimento por depressão, organizados em três pontos destacados na sequência.

\section{Aspectos da vida e do trabalho}

As mulheres entrevistadas apresentaram faixa etária entre 18 e 71 anos, com tempo de diagnósticos de depressão entre 2 e 22 anos, trabalharam no passado em pequenas propriedades rurais do município em estudo e, no período de coleta de dados desenvolviam atividades laborais diversas, a maioria delas com baixa escolaridade, sendo as principais ocupações relatadas: zeladora, cabelereira, ocupações em indústrias do ramo alimentício e, atividades domésticas como diaristas. 
O baixo grau de escolaridade pode ser compreendido pela necessidade que essas mulheres têm de trabalhar ao invés de estudar. $O$ trabalho familiar, seja na agricultura ou doméstico, se inicia ainda na infância, por determinação das condições sociais de pequenos agricultores. Desta forma, tem contato com agrotóxicos desde muito cedo e permanecem por anos expostas a essas substâncias.

[...] Eu comecei a trabalhar desde que eu me entendo por gente, eu era bem pequena [...] ia por que era obrigada, se não fosse apanhava do pai, mas eu via que não era certo e sempre dizia pro meu pai [...] nessa época eu estudei só até a sexta série, parei com 11 anos [...]. (E2)

Ah que eu me lembro foi desde bem novinha, eu tinha que ajudar com os trabalhos de casa [...] eu ajudava a minha mãe a cuidar dos meus irmãos mais novos, aí ajudava ela com os afazeres da casa mesmo, lavar roupas, limpar a casa, cozinhar. (E5)

\section{História de exposição e intoxicação por agrotóxicos}

A partir das narrativas, notou-se que o uso de agrotóxicos era comum e o contato acontecia de maneira intensa e, mesmo quando as lavouras eram cultivadas sem o uso desses produtos, o contato acontecia de maneira indireta através da dissipação de lavouras vizinhas.

[...] usava muito veneno, eu ajudava a preparar o produto, lavava as roupas da família, na hora da colheita também tinha o cheiro forte do veneno [...]. (E1)

[...] mas eu nunca tive contato, nem eu e nem o meu esposo. O que acontecia de vez em quando era só o cheiro [...] de outras lavouras, o que tinha veneno mesmo era o fumo que a gente mexia [...] só ajudava mesmo a carregar depois que já estava tudo certo. Então o contato próximo com o veneno eu nunca tive não. (E6)

Nossa queimava tudo, me dava muita tontura. Quando chegava em casa de noite, dava dor de estômago. (E2)

Uma vez eu me intoxiquei com esse veneno e eu me dei conta disso quando um dia eu passei 60 litros de veneno e era um dia de calor de rachar e aí meu filho me disse pra beber um pouco de água gelada e foi aí que eu passei mal, fiquei tonta e meus braços ardiam, por que eu não tinha lavado e eu sentia queimar, precisei ficar um dia deitada por que não aguentava minha cabeça e meu estômago doía [...]. (E5)

Quando questionadas sobre o uso de Equipamentos de Proteção Individual (EPIs), todas relataram que não utilizavam, pois em muitos casos, afirmaram que não tinham outras opções. Ressalta-se que as falas se remetem ao passado em que, possivelmente, 
não havia conhecimento sobre a importância do uso de EPIs, apesar de considerarem os produtos perigosos.

Naquele tempo acho que se chegasse com máscara todo mundo chamava de doido e falava que não precisava. (E4)

Não se usava nenhum tipo de proteção pra trabalhar, a gente sabia que era perigoso, mas ninguém usava nem luva, nem máscara, no máximo era uma camiseta de manga comprida. (E3)

\section{Adoecimento por depressão}

As entrevistadas relataram sintomas característicos relacionados à depressão em algum momento da vida: perda de interesse pela realização de atividades, baixa autoestima, falta de ar, episódios de choro frequentes, distúrbios do sono e desmaios. A partir do surgimento desses sintomas e os prejuízos na qualidade de vida buscaram ajuda médica e iniciaram o tratamento.

Ah eu me senti mal, me achava menor do que as outras pessoas, me sentia como um bicho que não tem valor, era mal cuidada por que trabalhava demais, era muito sol, me sentia velha e foi assim, pensava que era menor que todo mundo. [...] não tinha vontade de sair de casa, me escondia quando chegava alguém [...] além da dificuldade para dormir, eu tomo remédio até hoje. (E2)

Ah eu não sei explicar como é, mas são umas coisas ruins. Mas eu tinha dor de cabeça, falta de ar, esse tipo de coisa [...]. (E9)

\section{DISCUSSÃo}

As mulheres entrevistadas com diagnóstico de depressão eram adultas jovens, casadas, com baixa escolaridade e inseridas no mercado de trabalho conforme seu nível de instrução. Tais resultados não corroboram com estudo realizado com 1958 mulheres atendidas numa Unidade de Estratégia de Saúde da Família demonstrou que 28,5\% encontram-se na faixa etária entre 30 e 39 anos; 15,4\% não concluíram o ensino elementar; $54,5 \%$ não trabalham ou nunca trabalharam. Além disso, que fatores associados à ocorrência de depressão são possuir baixa escolaridade, trabalhar atualmente e ter doença mental prévia. $E$ fatores protetores como ser casada ou viver com companheiro, realizar atividades físicas regularmente e relatar autoavaliação positiva de saúde ${ }^{14}$.

Quando relacionamos o perfil de mulheres que tem como principal ocupação o trabalho no campo, e de mulheres que atuam fora da zona rural, notamos que as condições de vida e de trabalho são distintas. Em geral as trabalhadoras rurais estão expostas a riscos químicos, físicos e biológicos, sendo suas condições de saúde e trabalho 
mais precárias e consequentemente são mais vulneráveis ao surgimento de diversas doenças. As mulheres que atuam no meio urbano também estão expostas aos diversos agentes que podem ocasionar no surgimento de doenças, no entanto, geralmente são prestadoras de serviços de empresas sendo estas responsáveis pela saúde do seu trabalhador. No caso das trabalhadoras rurais, estas atuam diretamente nas suas propriedades e trabalham para obtenção do próprio lucro, sendo assim, permanecem desassistidas em relação à saúde e muitas vezes ultrapassam os limites biológicos para a manutenção de suas atividades ${ }^{15}$.

Nota-se no presente estudo que as participantes foram inseridas no trabalho ainda no período da infância. Em relação a isso, o Estatuto da Criança e do Adolescente proíbe qualquer trabalho a menores de quatorze anos de idade, salvo na condição de apren$d i z^{16}$. No entanto, o trabalho infantil na agricultura é percebido como uma ajuda à família, benéfico para a formação profissional, desenvolvimento moral e do caráter da criança. Sendo entendido como algo decorrente do interesse destes ou ainda da necessidade da família ${ }^{17}$. Deste modo a baixa escolaridade apresentada pelas entrevistadas apresenta-se como um fator de risco à saúde, considerando a dificuldade de entendimento, leitura e compreensão sobre informações apresentadas nas embalagens dos produtos utilizados, além das recomendações sobre a forma de utilização dos mesmos ${ }^{14}$.

Em relação à exposição aos agrotóxicos, as informações deste estudo mostram que as participantes referem não entrar em contato direto com tais substâncias, apesar disso, são elas que na maioria das vezes auxiliam na produção manipulando os produtos para aplicação ou lavando equipamentos e roupas que estiveram em contato direto com essas substâncias utilizadas no processo de produção ${ }^{14}$. No entanto, a Norma Regulamentadora $n^{\circ} 31$ define a exposição direta aos agrotóxicos a manipulação dos produtos desde o armazenamento, transporte, preparo, aplicação, descarte ou na lavagem de equipamentos e roupas utilizadas durante o processo de manipulação. A exposição indireta acontece pela circulação próxima a áreas de manipulação e por contato com resíduos encontrados em alimentos e na água ${ }^{18}$. A exposição aos agrotóxicos é considerada um problema de saúde pública, pois ao longo do tempo de exposição esses trabalhadores passam a desenvolver problemas de saúde agudos ou crônicos, e devido ao pouco conhecimento sobre os problemas ocasionados se faz necessário ações de conscientização, promoção e proteção à saúde desta população ${ }^{15}$.

Apesar da exposição prolongada gerar riscos e impactos para a saúde, os agrotóxicos são considerados pelos agricultores como essenciais para a produção como garantia do desenvolvimento da lavoura. Nesta situação, os agricultores passam a minimizar os riscos aos quais estão expostos, não encontrando saídas senão a incorporação desses produtos no processo de trabalho, já que não há incentivo governamental à incorporação de tecnologias alternativas no desenvolvimento da produção ${ }^{19}$. 
As formas de contato com agrotóxicos das entrevistadas foram, principalmente, por exposição indireta, através da dissipação dos produtos pelo ar e contaminação ambiental, sendo que duas entrevistadas relataram episódio de intoxicação aguda após manipulação direta.

As intoxicações causadas pelos agrotóxicos são classificadas em agudas, subagudas ou crônicas, de acordo com o tempo de exposição e os sintomas provocados ${ }^{2,6}$. Os sintomas relatados foram de intoxicação aguda: ardência na pele no momento da aplicação, tontura e cefaleia. Não foram relatados casos de intoxicações crônicas pelas entrevistadas, porém, os sintomas desse tipo de intoxicação são de difícil diagnóstico e podem surgir após meses ou anos do contato, e muitas vezes acabam não sendo relacionados no momento do diagnóstico?

A exposição intensa a agrotóxicos, demonstrada em anos de exposição ou intoxicações, também foi apontada como um fator que exerce influências sobre o aumento da prevalência do adoecimento mental ${ }^{20}$. Os trabalhadores expostos a essas substâncias muitas vezes não consideram a segurança no trabalho para atuar minimizando os danos.

Outra informação das participantes se refere ao não uso de EPI's. Sobre isso, entende-se que a utilização desses equipamentos se relaciona com o baixo nível de escolaridade e a dificuldade na compreensão dos rótulos e bulas dos produtos, a utilização de termos técnicos, informações confusas e o tamanho da fonte ${ }^{21}$. E grande parte dos trabalhadores rurais considera perigoso o uso de agrotóxicos, e que seu uso por período prolongado pode ocasionar no desenvolvimento de doenças e agravos à saúde, no entanto relacionam a importância do uso de EPI's apenas quando acontece casos de intoxicações, pois ainda há uma subestimação de que o uso de equipamentos de proteção possa evitar as intoxicações ${ }^{22}$.

Além da menor adesão ao uso de EPl's, a intoxicação crônica caracteriza-se por vários fatores, como determinantes relacionados ao produto e à pessoa exposta. Destaca-se a exposição ocupacional, a utilização de equipamentos de proteção individual e coletiva, a co-exposição a outros agentes tóxicos, a susceptibilidade genética, a idade, o sexo, os aspectos nutricionais e comportamentais, e os níveis de escolaridade ${ }^{23}$.

As intoxicações crônicas dão sinais ao longo do tempo, com o surgimento de sinais e sintomas com o decorrer do tempo de exposição aos agrotóxicos, deste modo se faz necessário a ação dos serviços de saúde sobre a conscientização da importância do uso de equipamentos de proteção, assim como de ações de promoção e proteção à saúde e treinamentos sobre o uso correto dessas substâncias, além disso, evidencia-se a necessidade de incentivo ao uso de tecnologias que possam diminuir o uso dos agrotóxicos, assim como preservar a saúde desta população ${ }^{22}$. 
Estudos indicam que a exposição prolongada a agrotóxicos pode estar relacionada ao desenvolvimento de doenças neurocomportamentais, entre elas a depressão. O contato com organofosforados inibe o processo de comunicação neuronal da acetilcolinesterase ocasionando o acúmulo desta enzima nos terminais sinápticos que pode provocar alterações clínicas que, em geral, são de difícil identificação, mas que pode estar relacionada a sérios danos à saúde do trabalhador agrícola, como a depressão grave e suicídio ${ }^{12,16,24}$.

Em contrapartida, estudos apontam que trabalhadores rurais que não utilizam agrotóxicos no processo de produção possuem melhor qualidade de vida e são menos propensos ao desenvolvimento de doenças, já os trabalhadores que utilizam tais substâncias são mais propensos ao desenvolvimento de doenças como a depressão, e até mesmo tentativas de suicídio ${ }^{15}$.

Intoxicações constantes e casos de envenenamento por agrotóxicos são mais fortemente relacionados à depressão e ideação suicida, além dos efeitos neurotóxicos relacionados a alterações nos níveis de serotonina e acetilcolina. Além disso, outro importante fator indicado quanto aos trabalhadores rurais é a falta de apoio social que agrava o sofrimento mental ${ }^{8}$.

Porém, deve-se ressaltar que a depressão, assim como outros transtornos mentais, são processos que resultam de múltiplas determinações sociais. Mulheres e pessoas com baixa escolaridade, o desemprego, as formas de trabalho com menor grau de qualificação, como as atividades domésticas, também foram relacionados ao adoecimento mental, os fatores desencadeadores estão associados à informalidade e instabilidade, assim como a baixa remuneração ${ }^{25-26}$.

Cabe destacar a dificuldade no estabelecimento de nexo causal entre as intoxicações e os problemas de saúde mental, em especial a depressão, e o autorrelato sobre tal condição pode ser considerado um fator de limitação a esse estudo, apesar de ser divulgado em muitos trabalhos científicos.

\section{CONCLUSÃO}

A partir da realização deste estudo foi possível descrever o perfil, os aspectos de vida e trabalho, a história de exposição e intoxicação por agrotóxicos e o adoecimento por depressão de mulheres do município em estudo.

O uso intenso de agrotóxicos pode ser considerado problema de saúde pública, pois contribui para o desenvolvimento de intoxicações agudas e condições crônicas como os transtornos mentais, especialmente a depressão e pode estar relacionado com outros processos de adoecimentos ainda em estudo. 
Nesse sentido, cabe aos serviços de saúde a investigação de casos crônicos de intoxicação e a notificação para que possam ser definidas políticas de saúde tanto para a atenção aos indivíduos expostos como para o controle da venda, utilização e descarte dessas substâncias.

O estudo apresenta como limitação o desenho metodológico, tendo em vista que investigou parte das mulheres acometidas por depressão e expostas a agrotóxicos em apenas um município. No entanto, não é limitado para descrever os aspectos vida e trabalho de mulheres acometidas por depressão em área rural que se propôs investigar.

Assim, entende-se que os resultados podem subsidiar estudos em outras realidades a fim de que se possa conhecer os impactos da exposição humana aos agrotóxicos para a saúde mental e contribuir para o fortalecimento de políticas públicas que visem a prevenção de agravos e promoção da saúde da população, assim como para subsidiar a formação em saúde e as práticas profissionais na atenção a essa clientela.

\section{REFERÊNCIAS}

1. Carneiro FF, Rigotto RM, Augusto LGS, Friedrich K, Búrigo AC (Org). Dossiê ABRASCO: um alerta sobre os impactos dos agrotóxicos na saúde. São Paulo: Expressão Popular; 2015. Disponível em: https:// www.abrasco.org.br/dossieagrotoxicos/wp-content/uploads/2013/10/DossieAbrasco_2015_web.pdf

2. IPARDES- Instituto Paranaense de Desenvolvimento Econômico e Social. Caderno Estatístico do Município de Anahy. Curitiba/PR; 2018.

3. ADAPAR. Agência de Defesa Agropecuária do Paraná. Utilização de agrotóxicos em quilograma no município de Anahy do Oeste. Curitiba/PR: ADAPAR; 2015

4. Brasil. Ministério da Saúde. Relatório: Vigilância em Saúde de Populações Expostas a Agrotóxicos no Estado do Paraná. Brasília: Ministério da Saúde; 2015. Disponível em: http://portalarquivos.saude.gov.br/ images/PDF/2015/julho/08/Relatorio-Paran--.pdf

Santos CA, Machado HC. O uso de Agrotóxicos e a Saúde do Trabalhador Rural- Seus Aspectos Comportamentais e Fisiológicos. Novos Direitos- Revista Acadêmica do Instituto de Ciências Jurídicas. 2015; 2: 117-126. Disponível em: https://revistas.unifan.edu.br/index.php/RevistalCJ/article/view/69/64

6. Paraná. Secretaria de Saúde do Estado do Paraná. Intoxicações agudas por agrotóxicos: atendimento inicial ao paciente intoxicado. Curitiba/ PR: Secretaria de Saúde do Estado do Paraná; 2018. Disponível em: https://www.saude.pr.gov.br/sites/default/arquivos_restritos/files/documento/2020-04/intoxicacoesagudasagrotoxicos2018.pdf

7. Bombardi LM. Geografia do uso de Agrotóxicos no Brasil e Conexões com a União EuropeiaSão Paulo: Laboratório de Geografia Agrária FFLCH- USP;2017. Disponível em: https://www.infraestruturameioambiente.sp.gov.br/educacaoambiental/prateleira-ambiental/geografia-do-uso-de-agrotoxicos-no-brasil-e-conexoes-com-a-uniao-europeia/

8. Joo Y, Roh S. Risk factors associated with depression and suicidal ideation in a rural population. Environ Health Toxicol. 2016;26;31:e2016018. Disponível em: https://doi.org/10.5620/eht.e2016018

9. Quandt AS, Chen H, Grzywacz JG, Vallejos QM, Galvan L, Arcury TA. Cholinesterase Depression and Its Association with Pesticide Exposure across Agricutural Season among Latino Farmworkers in North Carolina. Environ Health Perspect.2010; 118:635-639. Disponível em: https://doi.org/10.1289/ehp.0901492

10. Murakami Y, Pinto NF, Albuquerque GSC, Perna PO, Lacerda A. Intoxicação crônica por agrotóxicos em fumicultores. Saúde Debate. 2017;41:563-76. Disponível em: https://doi.org/10.1590/0103-1104201711317 
11. World Health Organization. World Health Organization. Depression and other common mental disorders: global health estimates. WHO; 2017. Disponível em: https://apps.who.int/iris/bitstream/handle/10665/254610/WHO-MSD-MER-2017.2-eng.pdf

12. Gangemi S, Miozzi E, Teodoro M, Briguglio G, De Luca A, Alibrando C, et al. Occupational exposure to pesticides as a possible risk factorfor the development of chronic diseases in humans (Review). Mol Med Rep. 2016; 14:4475-4488. Disponível em: https://doi.org/10.3892/mmr.2016.5817

13. Bardin L. Análise de Conteúdo. Lisboa: Edições 70,2004.

14. Gonçalves AMC, Teixeira BTM, Gama JRA, Lopes CS, Silva GA, Gamarra CJ, et al. Prevalência de depressão e fatores associados em mulheres atendidas pela Estratégia de Saúde da Família. J. bras. psiquiatr. 2018; 67: 101-109. Disponivel em: https://doi.org/10.1590/0047-2085000000192

15. Souza S, Pappen M, Krug SBF, Renner JDP, Reuter CP, Pohl HH. Uma revisão narrativa associando a vulnerabilidade à saúde e os fatores ambientais de trabalhadores rurais. Rev Bras Med Trab. 2018; 16:503-08. Disponível em: https://doi.org/10.5327/Z1679443520180250

16. Centro de Defesa dos Direitos da Criança e do Adolescente (CEDECA). Estatuto da Criança e do Adolescente. CEDECA;2017.

17. Riquinho DL, Hennington, EA. Sistema integrado de produção do tabaco: saúde, trabalho e condições de vida de trabalhadores rurais no Sul do Brasil. Cad. Saúde Pública. 2016; 32:2415. Disponível em: https://doi.org/10.1590/0102-311X00072415

18. Brasil. Ministério do Trabalho. NR 31- Segurança e Saúde no Trabalho na Agricultura, Pecuária Silvicultura, Exploração Florestal e Aquicultura. Brasília: Ministério do Trabalho; 2018.

19. Viero CM, Camponogara S, Cezar-Vaz MR, Costa VZ, Beck CLC. Sociedade de risco: o uso de agrotóxicos e implicações na saúde do trabalhador rural. Esc Anna Nery. 2016; 20:99-105. Disponível em: https:// doi.org/10.5935/1414-8145.20160014

20. Neto MGF, Andrade RD, Felden ÉPG. Trabalho na agricultura: possível associação entre intoxicação por agrotóxicos e depressão. Revista Perspect. Ciência e Saúde 2018; 3:69-82. Disponível em: http://sys. facos.edu.br/ojs/index.php/perspectiva/article/view/192/191

22. Ristow LP, Battisti IDE, Stumm EMF, Montagner SED. Fatores relacionados à saúde ocupacional de agricultores expostos à agrotóxicos. Saúde Soc. São Paulo. 2020; 29:1-11. Disponível em: https://doi. org/10.1590/S0104-12902020180984

23. Azevedo MFA, Rosa ACS, Alves SR, Larentis AL, Moreira MF, Teixeira LR, et al. Prevalência do tremor essencial em população exposta ocupacionalmente a agrotóxicos no estado do Rio de Janeiro. Rev Bras Neurol.2018; 54:10-15. Disponível em: https://revistas.ufrj.br/index.php/rbn/article/view/16934

24. Wahlbrinck MG, Bica JB, Rempel C. Percepção dos agricultores do município de Imigrante (RS) sobre os riscos da exposição a agrotóxicos. RBCIAMB. 2017;44: 71-84. Disponível em: https://doi.org/10.5327/ Z2176-947820170128

25. Serrano-Medina A, Ugalde-Lizárraga A, Bojorquez- Cuevas MS, Garnica-Ruiz J, González-Corral MA, Gárcia-Ledezma A, et al. Distúrbios Neuropsiquiátricos em Agricultores Associados à Exposição a Praguicidas Organofosforados em uma Aldeia Rural do Noroeste do México. IJERPH. 2019;16: 689.

26. Senicato C, Azevedo RCS, Barros MBA. Transtorno mental comum em mulheres adultas: identificando os segmentos mais vulneráveis. Ciênc. saúde coletiva. 2018; 23: 2543-54. Disponível em: https://doi. org/10.1590/1413-81232018238.13652016 